\title{
Triple primary malignancies of surface osteosarcoma of jaw, myelodysplastic syndrome and colorectal cancer as a second primary cancer detected by PET2-[ $\left[{ }^{18}\right.$ F $]$-fluoro-2-deoxy-D-glucose positron emission tomography: A case report
}

\author{
NOBUYUKI MARUYAMA ${ }^{1,2}$, KAZUHIDE NISHIHARA $^{1,2}$, TOSHIYUKI NAKASONE $^{2}$, \\ MASANAO SAIO ${ }^{3-5}$, TESSHO MARUYAMA ${ }^{1,2}$, IORI TEDOKON ${ }^{6}$, TETSUYA OHIRA ${ }^{7}$, FUMIKAZU NIMURA ${ }^{1}$, \\ AKIRA MATAYOSHI $^{2}$, KEN-NOSUKE KARUBE $^{8}$, NAOKI YOSHIMI $^{3,4}$ and AKIRA ARASAKI ${ }^{1,2}$ \\ ${ }^{1}$ Department of Oral and Maxillofacial Functional Rehabilitation, Graduate School of Medicine, University of the Ryukyus; \\ ${ }^{2}$ Department of Oral and Maxillofacial Surgery, University Hospital of the Ryukyus; ${ }^{3}$ Department of Pathology and Oncology, \\ Graduate School of Medicine, University of the Ryukyus; ${ }^{4}$ Department of Pathology, University Hospital of the Ryukyus, \\ Nishihara, Okinawa 903-0215; ${ }^{5}$ Department of Laboratory Sciences, Gunma University Graduate School of Health Sciences, \\ Gunma 371-8514; ${ }^{6}$ Division of Endocrinology, Diabetes and Metabolism, Hematology, Rheumatology (Second Department of \\ Internal Medicine), Graduate School of Medicine, University of the Ryukyus; ${ }^{7}$ Department of Endoscopy, University of \\ the Ryukyus; ${ }^{8}$ Department of Pathology and Cell Biology, Graduate School of Medicine,
}

University of the Ryukyus, Nishihara, Okinawa 903-0215, Japan

Received November 8, 2017; Accepted April 12, 2018

DOI: $10.3892 / 01.2018 .8594$

\begin{abstract}
Second primary malignancy (SPM) is a severe issue for cancer survivors, particularly for osteosarcoma (OS) survivors. To date, the associations between subsequent SPM and OS have been well reported. Hematogenic and solid malignancies tend to occur following OS treatment. Reportedly, 2-[ $\left.{ }^{18} \mathrm{~F}\right]$-fluoro-2-deoxy-D-glucose positron emission tomography (FDG-PET) is mainly used in OS patients for initial cancer staging, to evaluate the response of neoadjuvant chemotherapy, and when recurrence or metastasis is clinically suspected. The present case report describes a 70-year-old man diagnosed with three primary malignancies: jaw OS,
\end{abstract}

Correspondence to: Dr Tessho Maruyama, Department of Oral and Maxillofacial Functional Rehabilitation, Graduate School of Medicine, University of The Ryukyus, 207 Uehara, Nishihara, Okinawa 903-0215, Japan

E-mail: h075324@eve.u-ryukyu.ac.jp

Abbreviations: SPM, second primary malignancy; OS, osteosarcoma; FDG-PET, 2-[ ${ }^{18}$ F]-fluoro-2-deoxy-D-glucose positron emission tomography; PM, primary malignancy; MDS, myelodysplastic syndrome; CT, computed tomography; MRI, magnetic resonance imaging; SUVmax, maximum standardized uptake value

Key words: osteosarcoma, second primary, head and neck, oral, myelodysplastic syndrome, colorectal cancer, FDG-PET, multiple primary neoplasms myelodysplastic syndrome and colorectal adenocarcinoma. To the best of our knowledge, this combination of malignancies has not been reported previously. Until now, there is no specific protocol of postoperative FDG-PET for OS patients. Few studies have described OS follow-up methods; therefore, there is no consensus on proper follow-up methods. In the present case report, the colorectal early-stage SPM was observed, without any symptoms, by FDG-PET/computed tomography. To avoid overlooking solid SPMs, it is suggested that FDG-PET should be performed in the long-term follow-up of OS patients.

\section{Introduction}

Osteosarcoma (OS) is a terrible malignancy characterized by osteoblastic differentiation (1). The tumor affects a wide range of age groups (2). Recent improvements in the survival of patients with malignant cancers (including OS) may contribute to an increase in the incidence of second primary malignancies (SPMs) (3). SPM is a serious problem for the survivors of OS during the follow-up period after treatment (4). The relationship between subsequent SPM and OS has been well reported (4-7), and both hematogenic and solid malignancies tend to occur after OS treatment (7). In OS patients, 2- $\left[{ }^{18} \mathrm{~F}\right]$-fluoro-2-deoxy-D-glucose positron emission tomography (FDG-PET) is primarily used for initial cancer staging $(8,9)$, to evaluate the response of neoadjuvant chemotherapy (10-12), and when recurrence or metastasis is clinically suspected $(9,13,14)$.

To date, no accurate protocol of postoperative FDG-PET for OS patients has been reported, and there is uncertainty 
regarding the most appropriate methods for follow-up (15-17). In addition, no accurate protocol for detecting SPMs after OS treatment exists. SPM can occur anywhere in the body. Early-stage malignancy sometimes manifests no clinical symptoms; in contrast, advanced-stage malignancy generally indicates a poorer prognosis. Therefore, malignancies, including SPM, should be detected as early as the clinicians can. We report a case of a patient with triple primary malignancies (PMs): OS of the jaw, myelodysplastic syndrome (MDS), and adenocarcinoma of the colorectum. After follow-up FDG-PET/computed tomography (CT) was performed, rectal cancer was detected unexpectedly as no clinical symptoms had been observed.

\section{Case report}

A 70-year-old man was referred to the Department of Oral and Maxillofacial Surgery, University Hospital of the Ryukyus (Nishihara, Japan) in December 2015, for further evaluation of an oral mass. The patient had a 10-month history of swelling with gradual pain in the gums of his lower jaw. He had no previous malignancies and had never been exposed to ionizing radiation or been administered chemotherapy. He also had no history of family cancer syndrome; however, his mother had a history of kidney cancer. He was a smoker and drinker at the first visit. Written informed consent was obtained from the patient.

Incisional biopsy was performed in his previous clinic, and the lesion had previously been pathologically diagnosed as a spindle cell sarcoma highly suggestive of OS. For details, the histological findings of the initially diagnosed specimens revealed an irregular arrangement of spindle- or oval-shaped tumor cells, accompanying eosinophilic matrix suggesting osteoid formation. Most of the lesions showed mild atypia with rich osteoid formation (Fig. 1A). Conversely, tumor cells showing high-grade atypia with low osteoid formation existed in part (Fig. 1B). According to immunohistochemical examination, Ki-67 labeling index was 30-40\% (Fig. 1C). Tumor cells were positively stained for CD56 and were partially positive for smooth muscle actin. The cells were negatively stained for CD34, S100, or bc1-2.

Physical examination showed an elastic-hard, 3.5x2.0-cm mass of the right mandibular premolar gum (Fig. 2A). No lymphadenopathy was found in the neck region. Panorama $\mathrm{X}$-ray and subsequent contrast-enhanced CT scans from the head to chest and contrast-enhanced magnetic resonance imaging (MRI) of the head and neck were performed. A $3.5 \times 3.0 \times 2.5-\mathrm{cm}$ mass without bone resorption or infiltration was observed. CT scan showed new bone formation on the mandible surface (Fig. 2B and C). Contrast-enhanced fat-suppression T1-weighted MRI showed a high-signal mass around the mandible bone; however, no invasion to the bone was found (Fig. 2D). The signal of the bone marrow was considered as a slight bone marrow edema. No other lesion was detected in the neck, bones, or lungs by the above tests. Next, FDG-PET/CT and bone scintigraphy were evaluated to identify the OS staging and any other potential lesions in the whole body. FDG-PET showed increased FDG uptake in the surface of right mandible [maximum standardized uptake value $($ SUVmax $)=8.82$ ] (Fig. 3A). In contrast, no other FDG uptake was seen in the whole body. Bone scintigraphy showed abnormal bone intake on the mandibular surface, but no other sign was found. Based on the above findings, we diagnosed the disease as jaw surface OS without metastasis at the initial presentation. The patient was administered a four-drug preoperative regimen; that is, two cycles of cisplatin and doxorubicin, four cycles of high-dose methotrexate, and additional oral TS-1 for 7 days preoperatively. No preoperative radiotherapy was performed. Second, FDG-PET/CT was performed to evaluate the effect of chemotherapy, and the mass of the lower jaw shrank clinically and radiologically. The FDG uptake in the right mandible was decreased (SUVmax=5.66) (Fig. 3B). No other indications of lesions were detected in the whole body. Thrombocytopenia, resulting from administration of chemotherapy, was controlled by pegfilgrastim. Subsequently, the patient underwent segmental resection of the right mandible (that is, wide local resection) with reconstruction of a vascularized fibular graft and ipsilateral supraomohyoid neck dissection (April 2016). Histopathological examination revealed 'OS, post-therapeutic state', and the surgical margins were negative. Surgical materials obtained by segmental resection of the right mandible showed a lot of newly formed woven bone attached to the existing mandibular bone, and most of the osteocytes and tumor cells were dead due to chemotherapy. Namely, tumor showed characteristics of bone formation on the surface of mandibular bone (Fig. 4). No tumor cell was found in the bone marrow. According to these findings, the initially obtained biopsy, clinical and radiological findings, it was suggested that a part of tumor cells showed high-grade atypia, although most of the tumor mass showed abundant bone formation, which arose from the bone surface. Therefore, we considered two possible diagnoses: high-grade OS of the mandible or parosteal OS with partial dedifferentiation to high-grade OS (18). However, because of the death of tumor cells due to chemotherapy, we could not determine which diagnosis was correct. There was no indication of metastasis to the lymph nodes. Postoperative chemo/radiotherapy for OS was not performed. Following the surgery, the patient has not shown recurrence or metastasis of OS till the time of this writing.

On the other hand, MDS was found because of a hematoma of the jaw after the surgery, and thrombocytopenia after the surgery was found during the blood testing performed for postoperative follow-up. In spite of platelet transfusion, the thrombocytopenia continued. Therefore, an additional blood test, a bone marrow examination, and a chromosome analysis were performed one month after the surgery (May 2016). Wilms' tumor gene was positive ( 74 copies/ $\mu \mathrm{g}$ RNA) from the blood test. Histopathological examination of the bone marrow revealed that the marrow was hypercellular, and micromegakaryocytes were abnormally highly expressed in cluster of differentiation 41 staining. The fat-to-cell ratio in the marrow was approximately 2:1, and no OS cells were found. The chromosome analysis revealed a Y-chromosome deficiency. On the basis of the examination results, low-risk MDS (refractory cytopenia of unilineage dysplasia) was diagnosed. The patient was given only a packed red blood cells and platelets transfusion, and no other treatment has been required till the time of this writing.

Eight months after the surgery for OS, the patient complained that his lower jaw felt different. Local recurrence was suspected and contrast-enhanced CT was performed; however, no lesion 

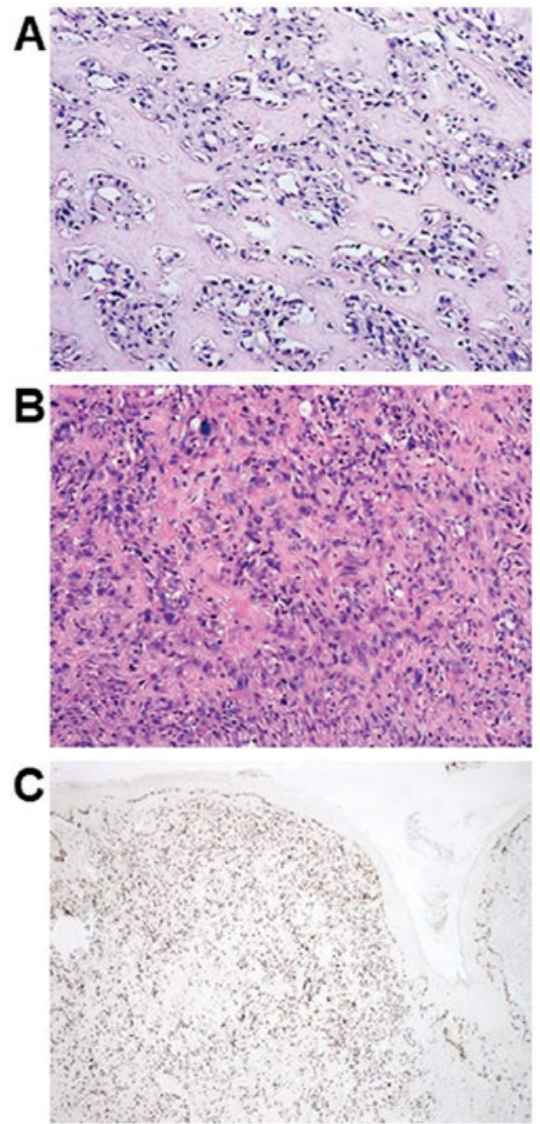

Figure 1. Histopathological examination revealed irregular arrangement of spindle- or oval-shaped tumor cells. In addition, osteoid lesions with an eosinophilic matrix in those cells were revealed. (A) The majority of the lesions were mild atypia with rich osteoid lesion (original magnification, x200); (B) Conversely, partially high-grade atypia with few osteoid lesions was observed (original magnification, x200). (C) Immunohistochemical examination for $\mathrm{Ki}-67$ determined that the labeling index was $30-40 \%$ (original magnification, $\mathrm{x} 40$ ).
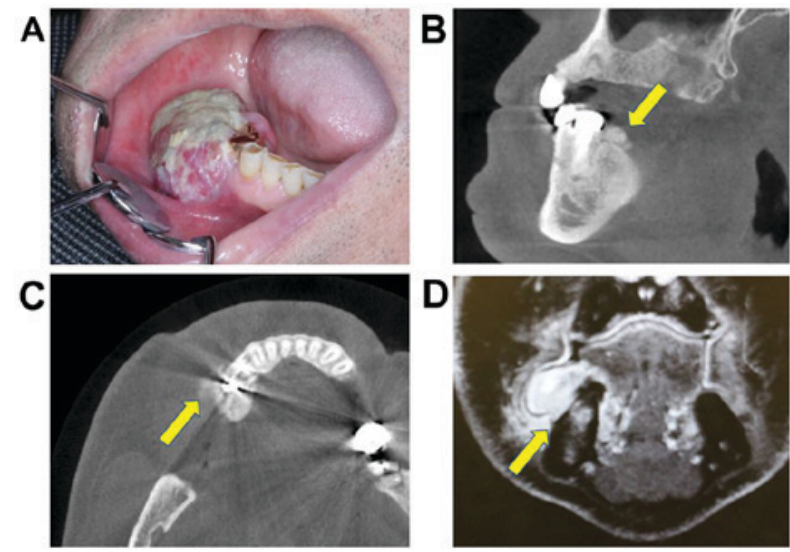

Figure 2. (A) Physical examination revealed an elastic, hard mass of the right mandibular premolar gum. A $3.5 \times 3.0 \times 2.5-\mathrm{cm}$ mass, without bone resorption or infiltration, was observed. (B and C) CT scan showed novel bone formation on the mandible surface. (D) Contrast-enhanced fat-suppression T1-weighted magnetic resonance imaging revealed the high-signal mass around the mandible bone; however, no invasion to the bone was identified. The signal of the bone marrow was considered as a slight bone marrow edema.

was found in the head and neck-to-chest region (range of the CT scan). Follow-up third FDG-PET/CT was performed 11 months
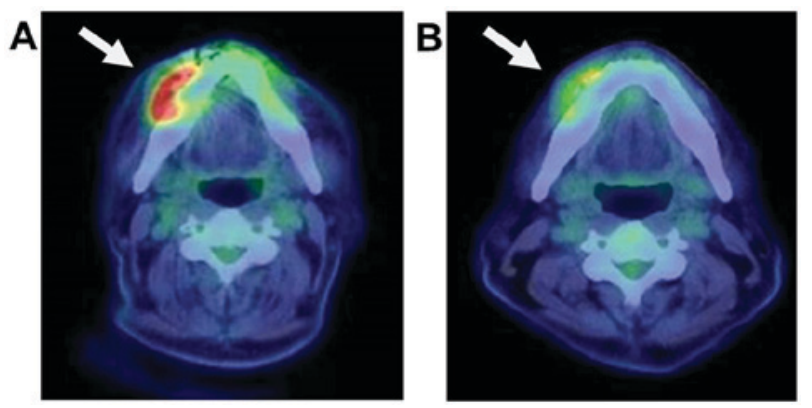

Figure 3. FDG-PET/CT and bone scintigraphy were performed to identify the osteosarcoma staging and any other lesions in the whole body. (A) FDG-PET showing increased FDG uptake in the right mandible (SUVmax=8.82; indicated by an arrow). (B) FDG-PET/CT for evaluating the effect of chemotherapy was performed and the mass of the lower jaw shrank radiologically. The FDG uptake in the right mandible was decreased (SUVmax=5.66; indicated by an arrow). FDG-PET, 2-[ $\left.{ }^{18} \mathrm{~F}\right]$-fluoro-2-deoxy-D-glucose positron emission tomography; CT, computed tomography; SUVmax, maximum standardized uptake value.

postoperatively, and abnormal uptake was detected in the rectum (SUVmax=14.58) (Fig. 5); on the other hand, no other lesion identified by uptake was found in the whole body, including the jaw, neck, bone, or lung. A colorectal tumor was suspected, and subsequent excision by endoscopic mucosal resection was performed (April 2017). Histopathological examination showed an adenocarcinoma and showed that the surgical margin was negative with no vascular invasion. On the other hand, due to the submucosal tumor invasion depth $(3,000 \mu \mathrm{m})$, the additional radical surgery has been considered. Based on the above, MDS and colorectal cancer were diagnosed after treatment of the jaw cancer. Five months after the resection of the rectal cancer (one year and five months after the jaw OS surgery), the patient was free of any malignant lesion. However, we plan to follow his progress carefully.

\section{Discussion}

This case highlights two important points: i) this combination of three PMs (jaw OS, MDS, and colorectal adenocarcinoma) has not been previously reported; and ii) to avoid overlooking solid SPMs, we suggest that FDG-PET should be performed in the long-term follow-up of OS patients.

Firstly, to our knowledge, there also have been no reports of cases involving both hematologic and solid malignancies after OS. We defined our case as involving three PMs by the Warren and Gates criteria reported in 1932 (19); that is, each malignancy was distinct and we excluded disease due to metastasis of one of the other malignancies. Using the criteria of Lee et al (7), we also discriminated between hematologic (leukemia, myeloproliferative disease, myelodysplastic disease, such as MDS, or lymphoma) and solid malignancies (all other malignancies). Thus, our patient had three PMs, including OS and subsequent SPMs involving hematologic and solid tumors. When we then searched PubMed and Google Scholar for English literature between 1932 and 2017, we found no cases describing the same combination of three malignancies, indicating the uniqueness of this case.

Patients with OS tend to develop both hematologic and solid SPMs after treatment (7). Compared with cancer-free 


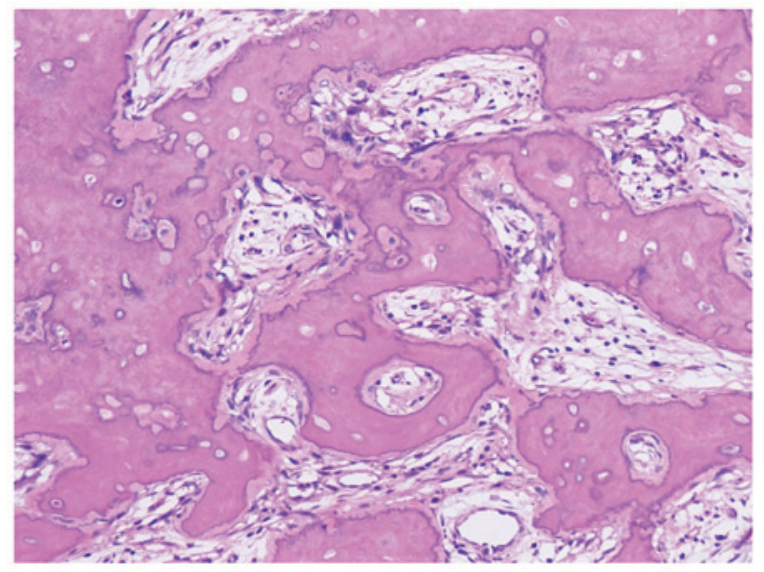

Figure 4. Histopathological examination revealed 'OS, post-therapeutic state', and the surgical margins were negative. A mass with irregular bone formation was observed. Degenerative tissues caused by the chemotherapy were identified in the mass; by contrast, no viable tumor cells were observed. The novel rich bone formation remained in the resected tumor, and the tumor was in contact with the mandibular bone (original magnification, x200). OS, osteosarcoma.

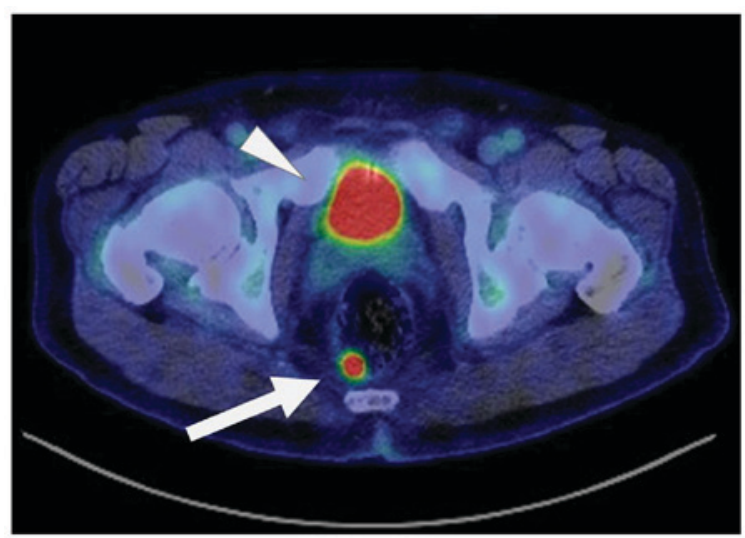

Figure 5. A total of 11 months following the treatment of jaw osteosarcoma, follow-up FDG-PET/computed tomography was performed, and an abnormal uptake was detected in the rectum (SUVmax=14.58; indicated by an arrow). In front of the uptake, physiological accumulation of the bladder was observed (indicated by an arrowhead) as the bladder is the major excretion route for FDG. FDG-PET, 2-[ $\left[{ }^{18} \mathrm{~F}\right]$-fluoro-2-deoxy-D-glucose positron emission tomography; SUVmax, maximum standardized uptake value.

individuals in the Childhood Cancer Survivor Study, survivors of OS tended to develop SPMs at a greater incidence (the standardized incidence ratio was 4.79) (20). Moreover, in a single-institution study, 26 of 1205 patients with OS of their extremities developed SPMs after treatment, which was significantly more frequent compared with the control group (1160 with benign tumors) (21). Cancer survivors are generally at risk of SPMs (22), with chemotherapy, radiotherapy, and a family history of cancer recognized to contribute to SPMs after OS (21,23-25). Our patient had received preoperative chemotherapy and his mother had a history of kidney cancer, but he had no history of family cancer syndrome (25). In addition, smoking and alcohol consumption are known independent risk factors of two of the malignancies in our patient (26-29). Further, old age is a risk factor for cancers such as colorectal cancer (30).
Other reports of triple to quintuple PMs involving OS as index malignancy $(21,24,31-33)$ (Table I) have been associated with intervals of 1-26 years between the diagnoses of OS and the SPMs (31-33), indicating that the occurrence of SPM, particularly solid malignancies, does not decrease over time, after OS treatment. Studies have generally reported that the average interval between OS and subsequent SPMs was 6.0-7.6 years $(5,7,21,24)$. Further, in large series, it has been shown that most SPMs occur $>10$ years after OS diagnosis, whereas most local or distant recurrences occur $\leq 5$ years, with as few as $5 \%$ of patients with OS developing their first local recurrence or distant metastasis greater than or equal to 5 years after initial treatment $(20,34)$. In a smaller study of OS survivors, the cumulative incidences of SPMs at 10, 20 , and 30 years were $2.1,4.0$, and $7.4 \%$, respectively, with solid malignancy developing at all times (7). Therefore, a long follow-up period is needed after primary treatment for OS to detect both SPMs as well as recurrence, metastasis, or multiple OS $(9,24,35,36)$. SPMs after OS can be fatal $(6,7)$, and together with metastasis, chemotherapy response, tumor characteristics, patient characteristics, surgical margins, and toxicity, SPMs are an important prognostic factor (37). Indeed, the prognosis is poor once SPMs occur (20). In these patients, given that advanced malignancy generally indicates a poorer prognosis, we recommend monitoring to detect SPMs early.

The surface OS of jaw is rare (38). Pathologically, we considered two possible histological diagnoses: high-grade surface OS and parosteal OS with partial dedifferentiation to high grade OS. Although the current case could not be clearly diagnosed due to tumor mass degeneration of surgical materials post chemotherapy. According to the findings of osteoid formation with tumor cell atypia showed in Fig. 1A-C, it was possible that the tumor was high-grade surface OS. However, the incidence of the high-grade surface OS at age 70 years was very unusual. Therefore, we should consider the possibility of parosteal OS with partial dedifferentiation to high-grade OS. Namely, all of the surface OSs of jaw tend to develop approximately 20-30 years old (39). Among those, parosteal OS occurs at a relatively higher rate in elderly patients, similar to our patient (38). In contrast, high-grade surface OS of jaw is very rare (38). Therefore, the current case may be parosteal OS with dedifferentiation of high-grade surface OS (40). In OS of the jaw (particularly the surface-type), metastasis is rare but can occur $(41,42)$. The most common site is the lung $(41,42)$. FDG-PET is a useful tool for detecting metastasis of OS (43). Conversely, SPM was detected by testing our patient.

Another issue is that FDG-PET should be performed during the long-term follow-up of OS to avoid overlooking solid SPMs. To date, the importance of detecting SPMs during follow-up after OS treatment has not been emphasized. In our case, MDS and colorectal cancer were metachronous according to Moertel's definition (i.e., recognized $\geq 6$ months after diagnosis) (44). MDS was easily diagnosed because of the hematoma observed after surgery and during the postoperative routine blood test (1 month after resection), which prompted early diagnostic bone marrow aspiration. By contrast, no clinical symptoms of colorectal cancer were present, and the tumor was found incidentally by FDG-PET/CT. Recent 
improvements in patient survival (including from OS) may lead to increased rates of SPMs (3). Further, old age is a risk factor of SPMs such as colorectal cancer (30). Therefore, PET/CT studies are recommended to screen for SPMs (22).

$\mathrm{PET} / \mathrm{CT}$ is useful for detecting SPMs in survivors of OS. However, they are not routinely included in OS follow-up protocols because few studies have used them for that purpose $(14,45)$. Indeed, FDG-PET tends to have been reserved for initial cancer staging and for evaluating the response of neoadjuvant chemotherapy (8-12), even though PET is especially useful after treatment for OS because it is not adversely affected by metal plates or implants (14). These scans can also detect three important disease patterns for which whole body examination is needed $(5,7,21,24,35,46)$ : i) recurrence or metastasis $(9,13,14)$; ii) multiple synchronous or metachronous OS $(35,46)$; and iii) synchronous or metachronous SPMs $(5,7,21,24)$. CT and MRI are inadequate because their coverage range is incomplete. Although OS is associated with a high incidence of lung metastases, indicating that chest CT is probably the best screening tool in most cases $(14,47)$, this can fail to identify SPMs at other sites. In the present case, we could not detect the rectal lesion by contrast-enhanced CT that was performed because local recurrence was suspected 8 months after OS treatment.

In daily practice, clinicians check local, regional, and distant sites where OS recurrences typically occur. Further, regarding SPMs for patients with head and neck cancers, they tend to recur locally in the head and neck, esophagus, or lung (48). SPMs at other sites (e.g., the colorectum) are relatively rare (49). In the current case, the early-stage colorectal SPM was found incidentally by FDG-PET/CT 11 months after treatment. OS is commonly associated with pain or swelling as an early symptom $(1,50)$, and recurrence may be suspected based on clinical symptoms (14). However, some SPMs are not associated with clinical symptoms, and it is known that early-stage colorectal cancer can be clinically silent (51). In practice, clinicians should consider the benefits and risks of performing FDG-PET/CT for patients after OS treatment. Although we have emphasized the benefits, the following are equally important considerations when choosing FDG-PET/CT: i) the test is generally expensive for patients (47); ii) physiological accumulation makes the detection of malignant lesions difficult, especially in the kidney or bladder (the major excretion route of FDG; Fig. 3); and iii) background activity may obscure the presence of lesions (52).

To date, no protocol exists which guides the use of FDG-PET after treatment for OS. Some studies have reported that follow-up PET/CT was useful even when there was no clinical evidence of recurrence or metastasis (14,53-56). However, no literature has emphasized the importance of detecting SPMs during follow-up for OS. We recommend that PET/CT should be performed during the follow-up of OS, specifically to detect SPMs.

In conclusion, the specific combination of triple PMs in this case (i.e., jaw OS, MDS, and colorectal adenocarcinoma) has not been reported previously. Based on our research, we recommend that FDG-PET be performed during the long-term follow-up of OS to avoid overlooking solid SPMs. However, our conclusions are based on a single case report, which limits their generalizability. Further cases are needed to help develop 
a protocol that describes the optimal role of FDG-PET or FDG-PET/CT scans in the identification of hidden synchronous or metachronous SPMs during the follow-up of patients with OS.

\section{Acknowledgements}

The authors would like to thank Dr. Hirofumi Matsumoto (Department of Pathology, University Hospital of the Ryukyus, Nishihara, Japan), for the pathological advice. The authors would also like to thank Ms. Chinatsu Toguchi and Ms. Ai Tokeshi (Department of Pathology, University Hospital of the Ryukyus) for their technical support.

\section{Funding}

No funding was received.

\section{Availability of data and materials}

All data generated or analyzed during this study are included in this published article.

\section{Authors' contributions}

NM and TM acquired the data, performed the literature review and edited the manuscript. AA substantially contributed to the concept and design of the study. KN, TN, IT, TO, FN and AM acquired the data and contributed clinical advice. MS, IT, TO and AA revised the manuscript. MS, KK and NY evaluated the specimens. MS gave histopathological advice. TM had a major role in writing the manuscript.

\section{Ethics approval and consent to participate}

The present case report was submitted for ethical review to the Ethics Committee of The University of the Ryukyus (Nishihara, Japan), which waived the requirement for review per institutional protocol as the case report does not contain content that requires ethical approval. The Ethics Committee approved the submission and publication of the manuscript. Written informed consent was obtained from the patient.

\section{Consent for publication}

Written informed consent was obtained from the patient for the publication of this case report and the accompanying images.

\section{Competing interests}

The authors declare that they have no competing interests.

\section{References}

1. Zhao L, Zhang J, Tan H, Wang W, Liu Y, Song R and Wang L: Gene function analysis in osteosarcoma based on microarray gene expression profiling. Int J Clin Exp Med 8: 10401-10410, 2015 .

2. Isakoff MS,Bielack SS, Meltzer P and Gorlick RL: Osteosarcoma: Current treatment and a collaborative pathway to success. J Clin Oncol 33: 3029-3035, 2015
3. Kim JS, Chung CY, Park HC, Myung DS, Cho SB, Lee WS, Min JJ and Joo YE: Synchronous quadruple primary tumors of thyroid, breast, pancreas and stomach: A case report. Anticancer Res 33: 2135-2138, 2013.

4. Aung L, Gorlick RG, Shi W, Thaler H, Shorter NA, Healey JH, Huvos AG and Meyers PA: Second malignant neoplasms in long-term survivors of osteosarcoma: Memorial sloan-kettering cancer center experience. Cancer 95: 1728-1734, 2002.

5. Pratt CB, Meyer WH, Luo X, Cain AM, Kaste SC, Pappo AS, Rao BN, Fleming ID and Jenkins JJ III: Second malignant neoplasms occuring in survivors of osteosarcoma. Cancer 80: 960-965, 1997.

6. Kim SH, Shin KH, Seok SO, Cho YJ, Noh JK, Suh JS and Yang WI: Secondary malignant neoplasms after osteosarcoma: Early onset and cumulative alkylating agent dose dependency. Ann Surg Oncol 22: 859-865, 2015.

7. Lee JS, DuBois SG, Boscardin WJ, Wustrack RL and Goldsby RE: Secondary malignant neoplasms among children, adolescents and young adults with osteosarcoma. Cancer 120: 3987-3993, 2014.

8. Quartuccio N, Treglia G, Salsano M, Mattoli MV, Muoio B, Piccardo A, Lopci E and Cistaro A: The role of Fluorine-18-Fluorodeoxyglucose positron emission tomography in staging and restaging of patients with osteosarcoma. Radiol Oncol 47: 97-102, 2013

9. Costelloe CM, Chuang $\mathrm{HH}$ and Daw NC: PET/CT of Osteosarcoma and ewing sarcoma. Semin Roentgenol 52: 255-268, 2017.

10. Harrison DJ, Parisi MT and Shulkin BL: The Role of 18F-FDG-PET/CT in pediatric sarcoma. Semin Nucl Med 47: 229-241, 2017.

11. Byun BH, Kong CB, Lim I, Choi CW, Song WS, Cho WH, Jeon DG, Koh JS, Lee SY and Lim SM: Combination of 18F-FDG PET/CT and diffusion-weighted MR imaging as a predictor of histologic response to neoadjuvant chemotherapy: Preliminary results in osteosarcoma. J Nucl Med 54: 1053-1059, 2013.

12. Byun BH, Kong CB,Lim I, Kim BI, Choi CW, Song WS, Cho WH, Jeon DG, Koh JS and Lim SM: Early response monitoring to neoadjuvant chemotherapy in osteosarcoma using sequential 18F-FDG PET/CT and MRI. Eur J Nucl Med Mol Imaging 41: $1553-1562,2014$

13. Hurley C, McCarville MB, Shulkin BL, Mao S, Wu J, Navid F, Daw NC, Pappo AS and Bishop MW: Comparison of (18) F-FDG-PET-CT and bone scintigraphy for evaluation of osseous metastases in newly diagnosed and recurrent osteosarcoma. Pediatr Blood Cancer 63: 1381-1386, 2016.

14. Angelini A, Ceci F, Castellucci P, Graziani T, Polverari G, Trovarelli G, Palmerini E, Ferrari S, Fanti S and Ruggieri P: The role of 18F-FDG PET/CT in the detection of osteosarcoma recurrence. Eur J Nucl Med Mol Imaging 44: 1712-1720, 2017.

15. Rothermundt C, Seddon BM, Dileo P, Strauss SJ, Coleman J, Briggs TW, Haile SR and Whelan JS: Follow-up practices for high-grade extremity Osteosarcoma. BMC Cancer 16: 301, 2016.

16. Paioli A, Rocca M, Cevolani L, Rimondi E, Vanel D, Palmerini E, Cesari M,Longhi A, Eraldo AM, Marchesi E, et al: Osteosarcoma follow-up: chest X-ray or computed tomography? Clin Sarcoma Res 7: 3, 2017.

17. Quartuccio N, Fox J, Kuk D, Wexler LH, Baldari S, Cistaro A and Schöder H: Pediatric bone sarcoma: Diagnostic performance of 18F-FDG PET/CT versus conventional imaging for initial staging and follow-up. AJR Am J Roentgenol 204: 153-160, 2015.

18. Lazar A and Mertens F: Parosteal osteosarcoma. In: WHO classification of tumors of soft tissue and bone. Fletcher CDM, Bridge JA, Hogendoorn PCW and Mertens F (eds). IARC Press, Lyon, pp292-293, 2013.

19. Warren S and Gates O: Multiple primary malignant tumors: a survey of the literature and a statistical study. Am J Cancer 16: 1358-1414, 1932.

20. Nagarajan R, Kamruzzaman A, Ness KK, Marchese VG, Sklar C, Mertens A, Yasui Y, Robison LL and Marina N: Twenty years of follow-up of survivors of childhood osteosarcoma: A report from the Childhood Cancer Survivor Study. Cancer 117: 625-634, 2011.

21. Bacci G, Ferrari C, Longhi A, Ferrari S, Forni C, Bacchini P, Palmerini E, Briccoli A, Pignotti E, Balladelli A, et al: Second malignant neoplasm in patients with osteosarcoma of the extremities treated with adjuvant and neoadjuvant chemotherapy. J Pediatr Hematol Oncol 28: 774-780, 2006. 
22. Almangush A, Coletta RD, Bello IO, Bitu C, Keski-Säntti H, Mäkinen LK, Kauppila JH, Pukkila M, Hagström J, Laranne J, et al: A simple novel prognostic model for early stage oral tongue cancer. Int J Oral Maxillofac Surg 44: 143-150, 2015

23. Bacci G, Ferrari S, Bertoni F, Ruggieri P, Picci P, Longhi A, Casadei R, Fabbri N, Forni C, Versari M and Campanacci M: Long-term outcome for patients with nonmetastatic osteosarcoma of the extremity treated at the istituto ortopedico rizzoli according to the istituto ortopedico rizzoli/osteosarcoma-2 protocol: An updated report. J Clin Oncol 18: 4016-4027, 2000.

24. Yonemoto T, Tatezaki S, Ishii T, Hagiwara Y and Inoue M: Multiple primary cancers in patients with osteosarcoma: Influence of anticancer drugs and genetic factors. Am J Clin Oncol 27: 220-224, 2004.

25. Hauben EI, Arends J, Vandenbroucke JP, van Asperen CJ, Van Marck E and Hogendoorn PC: Multiple primary malignancies in osteosarcoma patients. Incidence and predictive value of osteosarcoma subtype for cancer syndromes related with osteosarcoma. Eur J Hum Genet 11: 611-618, 2003.

26. Liu P, Holman CD, Jin J and Zhang M: Alcohol consumption and risk of myelodysplastic syndromes: A case-control study. Cancer Causes Control 27: 209-216, 2016.

27. Tong $\mathrm{H}, \mathrm{Hu} \mathrm{C}$, Yin $\mathrm{X}, \mathrm{Yu} \mathrm{M}$, Yang J and Jin J: A meta-analysis of the relationship between cigarette smoking and incidence of myelodysplastic syndromes. PLoS One 8: e67537, 2013.

28. Johnson CM, Wei C, Ensor JE, Smolenski DJ, Amos CI, Levin B and Berry DA: Meta-analyses of colorectal cancer risk factors. Cancer Causes Control 24: 1207-1222, 2013.

29. Parajuli R, Bjerkaas E, Tverdal A, Le Marchand L, Weiderpass E and Gram IT: Cigarette smoking and colorectal cancer mortality among 602,242 Norwegian males and females. Clin Epidemiol 7: $137-145,2014$

30. Siegel RL, Miller KD, Fedewa SA, Ahnen DJ, Meester RGS Barzi A and Jemal A: Colorectal cancer statistics, 2017. CA Cancer J Clin 67: 177-193, 2017.

31. Kubota M, Sawada M, Watanabe K, Koishi S, Kataoka A, Usami I, Lin YW, Okuda A, Akiyama Y and Furusho K Myelodysplastic syndrome presenting as third malignancy after non-Hodgkin's lymphoma and osteosarcoma. Ann Hematol 74 95-97, 1997.

32. Kimura K, Shinmura K, Hasegawa T, Beppu Y, Yokoyama R and Yokota J: Germline 553 mutation in a patient with multiple primary cancers. Jpn J Clin Oncol 31: 349-351, 2001.

33. Kousaka J, Fujii K, Yorozuya K, Mouri Y, Yoshida M, Nakano S, Fukutomi T, Takahashi E and Yokoi T: A case of quadruple primary malignancies including breast, tongue and thyroid cancers and osteosarcoma in a young female without karyotype abnormality. Breast Cancer 21: 500-503, 2014.

34. Hauben EI, Bielack S, Grimer R, Jundt G, Reichardt P, Sydes M, Taminiau AH and Hogendoorn PC: Clinico-histologic parameters of osteosarcoma patients with late relapse. Eur J Cancer 42: 460-466, 2006.

35. Jaffe N, Pearson P, Yasko AW, Lin P, Herzog C and Raymond K: Single and multiple metachronous osteosarcoma tumors after therapy. Cancer 98: 2457-2466, 2003.

36. Currall VA and Dixon JH: Synchronous multifocal osteosarcoma: Case report and Literature review. Sarcoma 2006: 53901, 2006.

37. Anderson ME: Update on survival in osteosarcoma. Orthop Clin North Am 47: 283-292, 2016.

38. van den Berg H, Schreuder WH and de Lange J: Osteosarcoma: A comparison of jaw versus nonjaw localizations and review of the literature. Sarcoma 2013: 316123, 2013.

39. Kumar VS, Barwar N and Khan SA: Surface osteosarcomas: Diagnosis, treatment and outcome. Indian J Orthop 48: 255-261, 2014.

40. Bertoni F, Bacchini P, Staals EL and Davidovitz P: Dedifferentiated parosteal osteosarcoma: The experience of the Rizzoli Institute. Cancer 103: 2373-2382, 2005.
41. Bertoni F, Dallera P, Bacchini P, Marchetti C and Campobassi A: The Istituto Rizzoli-Beretta experience with osteosarcoma of the jaw. Cancer 68: 1555-1563, 1991.

42. Sawair FA, Cheng J, Hao N, Maruyama S, Hoshina H, Takagi R, Koyama J, Hayashi T and Saku T: Periosteal osteosarcoma of the jaw bones: a clinicopathologic review. Oral Med Pathol 12: 3-10, 2007.

43. Brenner W, Bohuslavizki KH and Eary JF: PET imaging of osteosarcoma. J Nucl Med 44: 930-942, 2003.

44. Moertel CG, Dockerty MB and Baggenstoss AH: Multiple primary malignant neoplasms. I. Introduction and presentation of data. Cancer 14: 221-230, 1961.

45. Kim MS, Sim YS, Lee SY and Jeon DG: Occult thyroid carcinoma detected by FDG-PET scan in elderly osteosarcoma patients: Report of two cases. Ann Nucl Med 21: 529-532, 2007.

46. Gavane S, Price AP, Magnan H, Mahajan S and Pandit-Taskar N: Multifocal osteosarcoma: Unusual presentation and imaging findings. Clin Nucl Med 42: e202-e206, 2017.

47. Kneisl JS, Patt JC, Johnson JC and Zuger JH: Is PET useful in detecting occult nonpulmonary metastases in pediatric bone sarcomas? Clin Orthop Relat Res 450: 101-104, 2006.

48. Jain KS, Sikora AG, Baxi SS and Morris LG: Synchronous cancers in patients with head and neck cancer: Risks in the era of human papillomavirus-associated oropharyngeal cancer. Cancer 119: 1832-1837, 2013.

49. Coyte A, Morrison DS and McLoone P: Second primary cancer risk-the impact of applying different definitions of multiple primaries: Results from a retrospective population-based cancer registry study. BMC Cancer 14: 272, 2014.

50. Yildiz FR, Avci A, Dereci O, Erol B, Celasun B and Gunhan O: Gnathic osteosarcomas, experience of four institutions from Turkey. Int J Clin Exp Pathol 7: 2800-2808, 2014.

51. Kahi CJ, Imperiale TF, Juliar BE and Rex DK: Effect of screening colonoscopy on colorectal cancer incidence and mortality. Clin Gastroenterol Hepatol 7: 770-775, 2009.

52. Wang HY, Ding HJ, Chen JH, Chao CH, Lu YY, Lin WY and Kao CH: Meta-analysis of the diagnostic performance of [18F]FDG-PET and PET/CT in renal cell carcinoma. Cancer Imaging 12: 464-474, 2012.

53. Chang KJ, Kong CB, Cho WH, Jeon DG, Lee SY, Lim I and Lim SM: Usefulness of increased 18F-FDG uptake for detecting local recurrence in patients with extremity osteosarcoma treated with surgical resection and endoprosthetic replacement. Skeletal Radiol 44: 529-537, 2015.

54. Franzius C, Daldrup-Link HE, Wagner-Bohn A, Sciuk J, Heindel WL, Jürgens $\mathrm{H}$ and Schober O: FDG-PET for detection of recurrences from malignant primary bone tumors: Comparison with conventional imaging. Ann Oncol 13: 157-160, 2002.

55. Piperkova E, Mikhaeil M, Mousavi A, Libes R, Viejo-Rullan F, Lin H, Rosen G and Abdel-Dayem H: Impact of PET and CT in PET/CT studies for staging and evaluating treatment response in bone and soft tissue sarcomas. Clin Nucl Med 34: 146-150, 2009.

56. Fuglø HM, Jørgensen SM, Loft A, Hovgaard D and Petersen MM: The diagnostic and prognostic value of 18F-FDG PET/CT in the initial assessment of high-grade bone and soft tissue sarcoma. A retrospective study of 89 patients. Eur J Nucl Med Mol Imaging 39: 1416-1424, 2012.

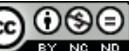

This work is licensed under a Creative Commons Attribution-NonCommercial-NoDerivatives 4.0 International (CC BY-NC-ND 4.0) License. 